\title{
Parameter Experimentation for Epileptic Seizure Detection in EEG Signals using Short-Time Fourier Transform
}

\author{
Ricardo Ramos-Aguilar ${ }^{1}$, J. Arturo Olvera-López ${ }^{1}$, Ivan Olmos-Pineda ${ }^{1}$, \\ Susana Snchez-Urrieta ${ }^{2}$, Manuel Martín-Ortiz ${ }^{3}$ \\ 1 Autonomous University of Puebla, Faculty of Computer Science, Puebla, Mexico \\ ricramosa1@gmail.com, \{aolvera,iolmos\}@cs.buap.mx \\ 2 Autonomous University of Puebla, Faculty of Electronics Science, Puebla, Mexico \\ susana.sanchezu@correo.buap.mx \\ 3 Autonomous University of Puebla, \\ Information Technology and Communications Department(DCyTIC), \\ National Laboratory of Supercomputing (LNS-BUAP-CONACYT), Puebla, Mexico \\ manuel.martin@correo.buap.mx
}

\begin{abstract}
Short-time Fourier transform is a time-frequency method commonly used to analyze signals, particularly in EEGs. It has shown acceptable results for the identification of different actions, such as sleep disorders, epilepsy, and others, and in applications as brain-computer interfaces. However, the selection of short time Fourier transform parameters is not a trivial task, as the variability of these directly affects the resolution spectrogram, from which features are extracted to determine the constructed models in the classification stage. In this paper, experiments for determining STFT parameters such as window type and length, and overlapping are explored. As a case study, an EEG epilepsy database is used to identify healthy people versus patients suffering epileptic seizures, finding that the parameters modify the spectrogram visualization in terms of time/frequency and classification. Based on these experiments, it was concluded that the proposed strategy supports the correct selection of parameters that positively impact the accuracy of the results obtained.
\end{abstract}

Keywords: EEG Signals, Short-time Fourier Transform, Spectrograms, Seizure Classification.

\section{Introduction}

Electroencephalography (EEG) is a brain monitoring method based on measurements of electrical activity generated by the brain. An EEG shows evidence of how the brain performs the bodily functions over time, such as the pumping of the heart, gland secretion, breathing, and internal temperature regulation, among others [10]. Nowadays, EEGs are used by scientists and physicians for 
analyzing brain functions and diagnosing neurological disorders, such as brain tumors, head injuries, sleep disorders, dementia, epilepsy, Alzheimer's, seizure disorder, deficit attention disorder, anxiety disorder, fetal alcohol syndrome, autism, as well as monitoring the effects of anesthesia during surgery $[3,5,6,8,16]$. The EEG is an appropriate tool to aid in the diagnosis of diseases, epilepsy in particular. This inexpensive tool is useful for showing the underlying manifestation of epilepsy. EEG signals in people with epilepsy show two kinds of abnormal activity: ictal (during an epileptic seizure) and interictal (between seizures) [8, 16]. EEG analysis for diagnosing epilepsy started in 1970, and since then it has been an area of interest for researchers due to its non-stationary features; at present, most problems in seizure detection are related to finding events (ictal and interictal) during epileptic seizures [8].

Different methodologies have been proposed to identify epileptic seizures in EEG signals based on frequency, time, wavelet transforms, and Gabor filters $[7,8,14]$. However, the nature of the EEG signals (non-stationary) involves specific aspects when employing techniques based on frequency or time [8], as the features obtained from these methods do not provide enough information from EEG signals alone [1]. Techniques based on Time-frequency analysis are considered more complete than some others because they decompose the signal in frequencies over signal time, to analyze non-stationary signals such as EEGs. Time-frequency representations can be applied to EEG signals, as the resulting transform can be treated as an image in order to extract features, which has shown acceptable results in terms of accuracy for different applications [13]. Short-Time Fourier Transform (STFT) is a time-frequency representation commonly used in signal analysis, as well as in digital image processing, voice processing, biology, medicine [9], and in other applications, such as Electroencephalographic (EEG) signals analysis (STFT allows a representation in time-frequency of the EEGs, which shows a different visualization for analysis).

From EEG spectrograms different features can be extracted to identify several tasks (imagined writing, motor images, epilepsy, alcoholism, etc.) [12]. In most of these, the STFT parameters are pre-defined by the programming language toolkits when beginning the analysis. Parameter selection is important, as it helps to minimize two problems: poor spectrogram resolution and a dearth of relevant features, which determine the results in the classification; in addition, the process of assigning specific parameter values for any application is a difficult task. Thus, different STFT parameters are analyzed in this work in order to identify relevant features that support the classification of epilepsy stages (seizure and normal) and their spectrograms. Initially, EEG signals are obtained from a dataset and STFT is applied with different parameters; after that, an image generated from the spectrogram is converted to grayscale to extract features; total energy and spectral peaks are obtained for training three different classifiers and evaluating STFT parameters; the results are shown in terms of the accuracy in binary classification and the visualization of some spectrograms. The STFT parameters of the experimentation were selected from main and side lobes of the windows; first, the width of the main lobe was analysed and the windows with 
Parameter Experimentation for Epileptic Seizure Detection in EEG Signals using Short-Time...

the thinnest were chosen, after the side lobes were observed and from its height with respect to the main lobe were selected the windows with side lobes lowest. The overlapping is proposed from window properties, where are selected in the case of lobes are added or attenuated when windows are overlapped. This paper is organized as follows: section 2 introduces the methodology and the related theory; section 3 provides a description of EEG dataset and the experimental analysis; Finally, section 4 presents our conclusions and future work.

\section{Methodology}

\subsection{Short-time Fourier Transform}

In practical applications, STFT is implemented as a sliding window adjusted to a signal. Given an input signal $x^{T}$ of arbitrary duration, segments are extracted at regular intervals using a time-limited window $w_{n}$; The segments (frames) of the signal can be expressed as [4]:

$$
x_{l}[n]=w_{n} * x[n+l L], \quad 0 \leq n \leq N-1,
$$

where $N$ is the length of the window, $l$ is the frame index, and $L$ is the hop size, that is, the sample spacing between consecutive applications of the sliding extraction window; the index $n$ is a local time index relative to the beginning of the window displacement. The expression $x[n+l L]$ represents a position over signal, $n$ is representing the phase to shift the window, $*$ represents a operation likewise modulation between two terms. Finally, the discrete Fourier Transform (DFT) [4] is applied to each frame of the signal, as follows:

$$
X[k, l]=\sum_{n=0}^{N-1} x_{l}[n] e^{-i 2 \pi n k / K}=\sum_{n=0}^{N-1} w_{n} * x[n+l L] e^{-i 2 \pi n k / K},
$$

where $K$ is the size of the DFT and $k$ is a frequency index or bin index. The STFT $X[k, l]$ then characterizes the local time-frequency behavior of the signal around time $l L$ and bin $k$; for a sampling rate of $F_{s}$, these discrete indices correspond to the continuous time $l L / F_{s}$ and frequency $k F_{s} / K[4]$. To simplify the notation, a radial frequency of:

$$
\omega_{k}=2 \pi k / K
$$

is frequently included; then, the expression STFT becomes:

$$
X[k, l]=\sum_{n=0}^{N-1} w_{n} * x[n+l L] e^{-i \omega_{k} n} .
$$

Applying the window over time, the STFT returns a spectral representation of a time segment of the input signal; interpreting $X[k, l]$ as a function of the frequency $k$ for each value of the time index $l$, the STFT corresponds to some series of spectra located in time. Alternatively, the STFT can be seen as a time 
function for each frequency; interpreting $X[k, l]$ as a time serie that is a function of $l$ for each bin $k$ (points to calculate the DFT), the STFT corresponds to a filter bank that decomposes the input signal over frequency channels or subbands [4]. These two interpretations of the STFT are represented with respect to the time-frequency plane. These changes to time and frequency have become common in the modern literature on signal processing as a way of representing the time and frequency resolutions of signals.

One drawback of STFT is the resolution required to obtain spectrograms, caused mainly by the length of the window. A narrow window offers a better resolution in time, but not in frequency, while a wide window provides good resolution in frequency, but does not perform as well in time resolution. This means that it is difficult to achieve a good location in both time and frequency domains simultaneously, because the STFT depends on only one window.

The STFT process is a method that begins by windowing a signal into shorter segments, where phase windows can be overlapped; Fast Fourier transform (FFT) is applied to each segment separately [15] and each result is joined to form the spectrogram, which can be manipulated, and then the inverse FFT is applied to return each segment to the time domain.

Therefore, to apply the STFT to signals, different parameters should be considered; for example: window type and length, main lobe length (corresponding to window type), FFT points and overlapping. These parameters that can affect the resolution spectrogram are briefly described in the following subsections.

\section{$2.2 \quad$ Windowing}

Windowing is a phase that involves applying a function to a signal or segment over time, which is then used in the Fourier transform or the STFT. The Fourier transform is commonly implemented for the analysis of non-periodic and stationary signals; for non-stationary signals, one option is STFT, which analyzes the signal by segments, making use of the windows, since this sub-signal is supposed to be a stationary signal. A good analysis depends essentially on the type of window and the parameters that can be modified, which will be discussed later.

First, the window type is selected; Figure 1 (a) shows different windows (Bartlett, Blackman, Hamming, Gaussian, Kaiser, rectangular, Hanning) with a length of 64 points and their spectra (b); these windows are the most common in the signal analysis literature. Initially, when a window is applied to the signal, it is modulated as the window way along time segment; the rectangular window is the ideal in time, since signals are not modified when it is applied and with this, it would not adjust to the window type due to the shape as a box, which only cut the signal and Fourier transform is applied. This window has a thin main lobe; although, its secondary lobes are very high. The main idea is to have something balanced, and the Gaussian window is optimal due to its very similar wave form spectrum; however, it cannot be obtained computationally, so approximations are implemented that show a main lobe that is average in terms of width, although the secondary lobes are not the smallest. The Blackman window shows the smallest secondary lobes, followed by Hanning, which has a small 
Parameter Experimentation for Epileptic Seizure Detection in EEG Signals using Short-Time...

main lobe in its window; this window is also a variant of the raised cosine, which is very similar in both spectrum and window. Taking into account the windows in Figure 1, it can be seen that the main lobes are in ascending order by window length: Blackman, Gaussian, Hamming, Hanning, Kaiser and Rectangular; the secondary lobes are listed in relation to height (from highest to lowest energy): Rectangular, Kaiser, Hamming, Gaussian, Hanning, and Blackman.

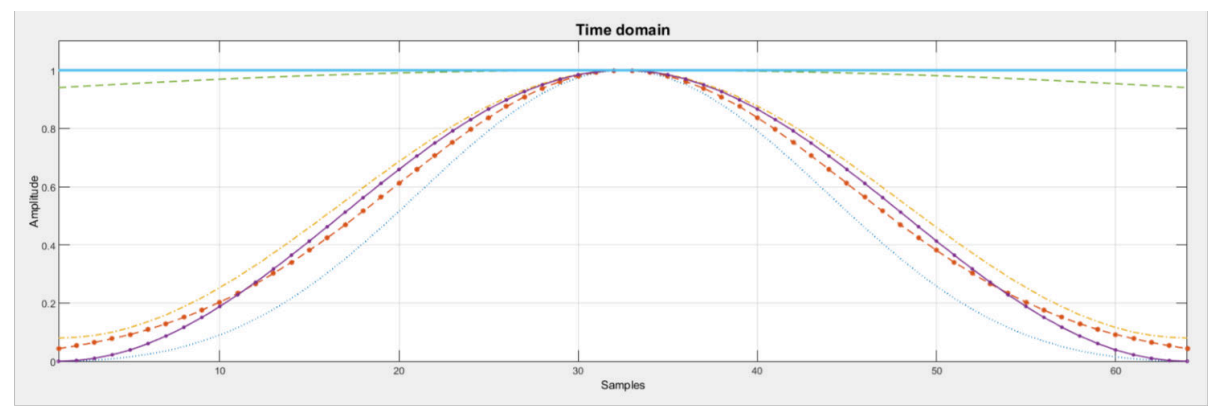

(a)

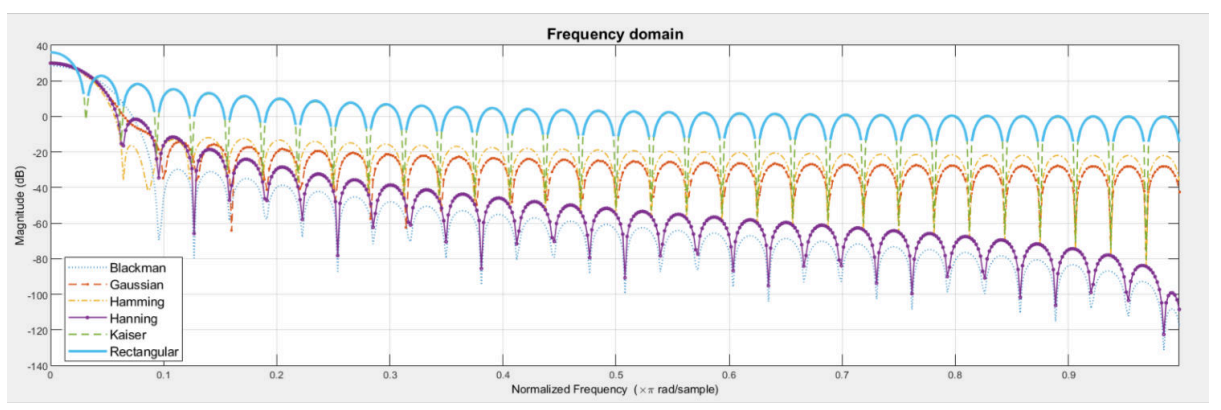

(b)

Fig. 1. Windows (a): (....)-Blackman, (..-.)-Gaussian, (-.-.)-Hamming, (-.-)-Hanning, (_ _ - )-Kaiser, and (_-)-Rectangular, 64-point length and their spectra(b).

On the other hand, temporal and frequency resolution can be obtained from the STFT parameters; Figure 1 shows the relationship between these parameters, where it can be seen that the spectrum is narrow for a wide rectangular window, so its use is not advisable, while Hamming and Blackman maintain a less strict relationship, since proportionally, it is not noticeable as in a rectangular window; this is due to both windows being variants of a raised cosine and to the lobes for both being smaller.

Considering a visual analysis from Figure 1 with main lobes and secondary side lobes as main features, any window can be selected as an acceptable option, because a thinner lobe shows frequencies close to that signal. In addition, the secondary lobes show a lower energy dispersion, as most are concentrated in 
the main lobe; however, these are not the only parameters to be considered in window analysis [11], although, a method based on spectral peaks might suffice; therefore, one parameter that defines spectral peaks is the main lobe of the windows, which represents peaks, and another is the secondary lobe height, because energy is disperse among these. Thus, considering these two parameters, the selected windows were: rectangular, which has a representation similar to the Fourier Transform, as it does not have modification in time and its spectrum has the thinnest main lobe; Gaussian, with a window similar to the spectrum; Blackman, because it has the lowest secondary lobes; and Hanning, because it has one thin main lobe and low secondary lobes.

\subsection{Feature Extraction}

In this work, features are obtained from EEG signal spectrograms. Spectral peaks are found first, since these are related to relevant energy over some frequency/time; after that, different features are extracted from the peaks. Finally, volume features are calculated from all intensities of the spectrogram.

Spectral peaks. These peaks are calculated using the local maxima method, which find local minima within a region and above a threshold from energy levels, these are represented on the spectrogram. From a region or mask the peaks is found with the method mentioned. Up to this point, other parameters have not been analyzed, since these peaks depend on the window and the signal type, which will be analyzed later.

Volume. Feature extraction is based on an approach that considers the spectrogram in a three-dimensional representation as a volume. All intensity points and time and frequency coordinates were used to obtain the spectrogram volume. Likewise, all intensities were added together to obtain the approximate energy. The spectral peaks were used to calculate the surface area and the intensities from the highest peaks were added; the area formed by peaks and volume from all spectrogram points were calculated through a convex hull. Figure 2 shows these five features, which are concatenated to obtain the feature vector.

\section{Results}

\subsection{Experimental Approach}

This section presents an evaluation of STFT parameters such as overlapping and window type and length. These experiments are concerned with the identification of people experiencing epileptic seizures or healthy people. First, signals were obtained from an EEG dataset (epileptic seizure); STFT with different windows and overlapping $(0 \%$ and $75 \%$ ) was applied to each signal to obtain spectrograms, which were converted to grayscale images; after that, peaks were found from 


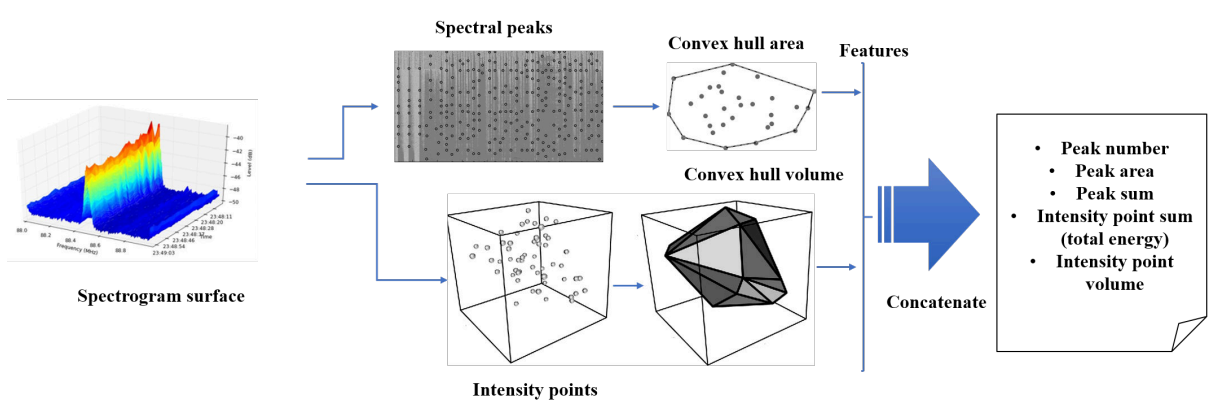

Fig. 2. Features extracted form EEG signal: peak number, peak area, peak sum, intensity point sum and intensity point volume.

regions, these are shaped by 2 xminimaldistance +1 , where minimaldistance between peaks is the separation between peaks; to find the maximum number of peaks the value of 1 was used as a separation distance; a threshold was selected from the minimum intensity of the spectrogram in grayscale. The features describe below were extracted from the peaks.

For the experiments, four windows were taken into account, with Gaussian being the closest to the ideal, the rectangular being the ideal in time, Blackman having the smallest lobes, and Hanning maintaining the best relationship between the minimum width of the main lobe and the height of the secondary lobes. The number of FFT points are the same for the window length, which is not relevant for resolution; a $75 \%$ overlapping was proposed, based on the properties of the windows and their spectral responses.

This methodology was tested using an open access epilepsy EEG dataset from Bonn University [2] with five subsets (Z,O,N,F, and S), each subset containing 100 EEG signals with 4097 samples, recorded at a sampling rate of $173.61 \mathrm{~Hz}$ using a 128-channel amplifier system with an average common reference. Sets Z and $\mathrm{O}$ were collected from five healthy volunteers. Sets N, F, and $\mathrm{S}$ were recorded from five epileptic patients for each set. Records from set $\mathrm{S}$ were collected during seizure activity, while sets $\mathrm{N}$ and $\mathrm{F}$ were gathered during seizure-free intervals.

From the epileptic dataset, two subsets (subset A and E) were used to classify the signals, which correspond to healthy people and patients suffering an attack, respectively. Firstly, the signals from healthy people (Figure 3) were analyzed using different STFT parameters.

STFT was applied to EEG signals from subset A, as mentioned above, and four windows with length (64) and without overlapping were used. The spectrograms generated are shown in Figure 4, which describes scattered energy, because there are different events and frequencies over time; also, the spectrogram maintains its relationship with the corresponding spectrum. The energy is more scattered along the vertical axis (frequency), while it is more noticeable in (b), due to the width of the main lobe of Gaussian spectrum and the energy in (a) not being as noticeable, since it has the narrowest main lobe (Rectangular). 


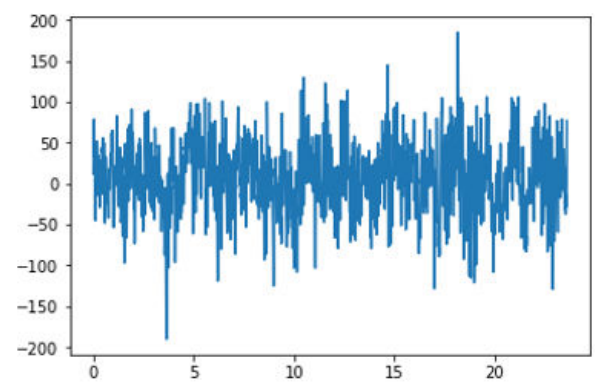

Fig. 3. EEG signal from an open eyes person in a relaxed state.
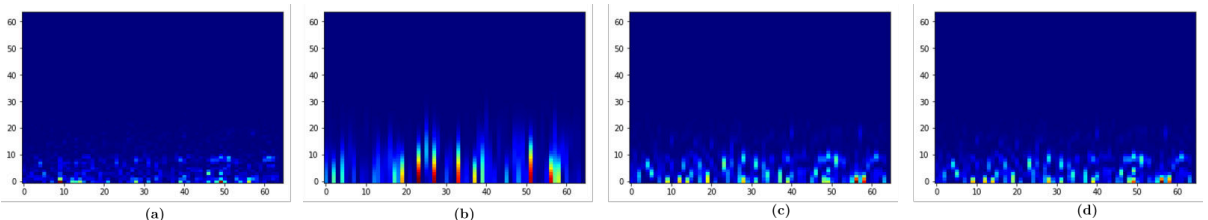

Fig. 4. Spectrogram from EEG signals of a healthy person in a relaxed state with eyes open. Window length $=64,0 \%$ overlapping: (a) Rectangular, (b) Gaussian , (c) Blackman and (d) Hanning.

Comparing the spectra from Figures 4 and 5, only an elongation of the energy on the horizontal axis (time) can be noted, although the windows are actually focusing more on the frequencies shown along the vertical axis. Something similar occurs when a single window is placed over the entire time from a signal: it shows only one frequency; otherwise, when a narrow window is applied to short segments from a signal, it shows changes over time, i.e., frequencies for each window.

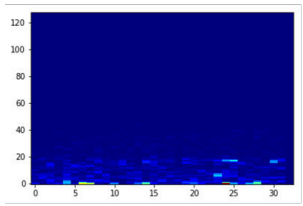

(a)

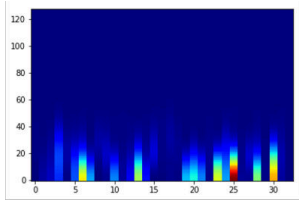

(b)

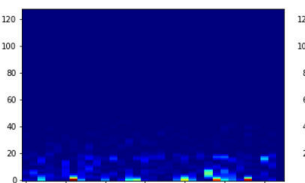

(c)

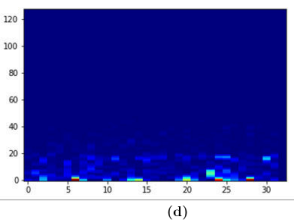

Fig. 5. Spectrogram from EEG signals from a healthy person in a relaxed state with eyes open. Windows length $=128,0 \%$ overlapping: (a) Rectangular, (b) Gaussian , (c) Blackman and (d) Hanning.

Another interesting parameter is overlapping, which implies a more general approach to the signal. When a high percentage of overlapping is applied, window 
$n$ overlaps most of window $n+1$, which could generate windows without side lobes (as they cancel each other out); only windows with overlapping main lobes cause the entire signal to resemble the application of a single window to the entire signal. Of the eight spectra, Figures 6 and 7 are most similar to those shown in Figures 4 and 5, but the overlap thins the energy shown over time.

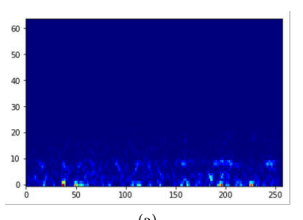

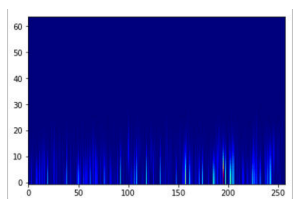

(b)

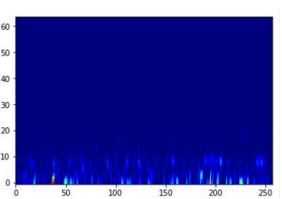

(c)

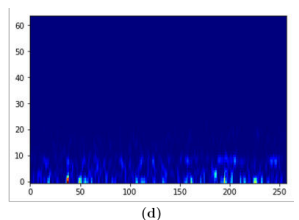

Fig. 6. Spectrogram from EEG signals from a healthy person in a relaxed state with eyes open. Window length $=64,75 \%$ overlapping: (a) Rectangular, (b) Gaussian , (c) Blackman, and (d) Hanning.

When the overlap is greater, the energy seems to be reduced along the $\mathrm{x}$-axis (time), and in terms of time-frequency resolution, a large window focused for the frequency would have a better resolution with a higher percentage of overlap.

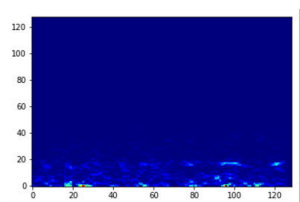

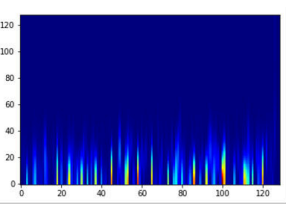

(b)

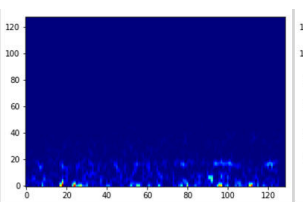

(c)

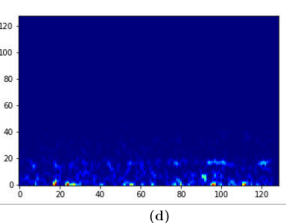

Fig. 7. Spectrogram from EEG signals from a healthy person in a relaxed state with eyes open. Window length $=128,75 \%$ overlapping: (a) Rectangular, (b) Gaussian , (c) Blackman, and (d) Hanning.

Signals from subset E were also analyzed, which correspond to patients suffering an epileptic seizure. The signal in Figure 8 represents an EEG of a person suffering an attack; as in previous experiments, the same windows were used.

The higher-frequency spectrograms in Figures 9-12 show higher energy for some sections in comparison to the spectrograms of a healthy person. This representation is similar to both kinds of EEG signals, due to the parameters used in these experiments. From the spectrograms of healthy people, energy activity appears along time in terms of energy, possibly due to some event occurring in different time intervals; this could be noted using a 64 Gaussian window. In the opposite case, for epileptic patients, the energy is concentrated along time, showing the energy as frequency by applying rectangular, Blackman, and Hanning windows. The Gaussian window describes activity in time, and can be appre- 
Ricardo Ramos-Aguilar, J. Arturo Olvera-López, Ivan Olmos-Pineda, Susana Sánchez-Urrieta, et al.

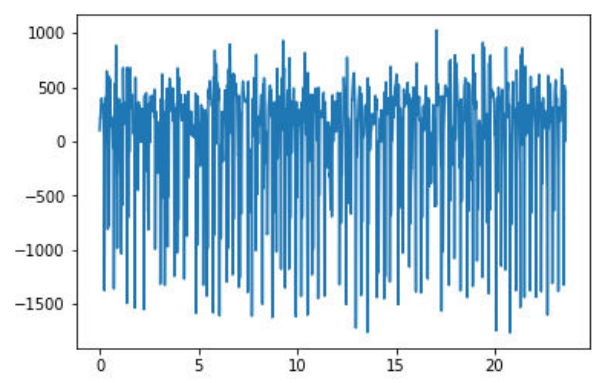

Fig. 8. EEG signal from a person suffering an epileptic seizure.

ciated when using long windows (128), for example, the window used (128) in Figure 12 with a $75 \%$ overlap.

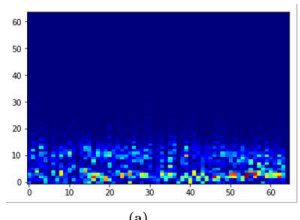

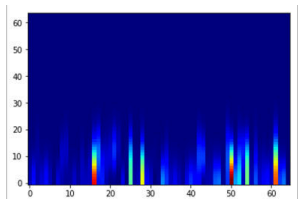

(b)

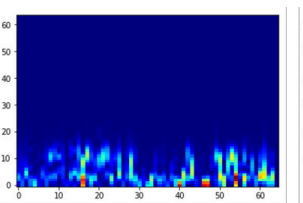

(c)

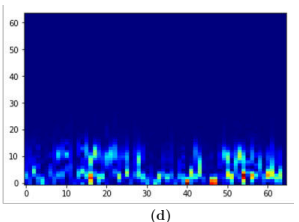

Fig. 9. Spectrograms of EEG signals from a person suffering an epileptic seizure. Window length $=64$ and $0 \%$ overlapping(a) Rectangular, (b) Gaussian , (c) Blackman and (d) Hanning.

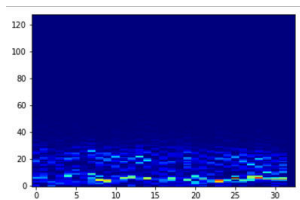

(a)

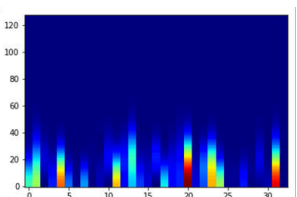

(b)

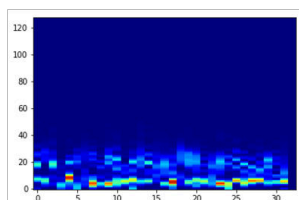

(c)

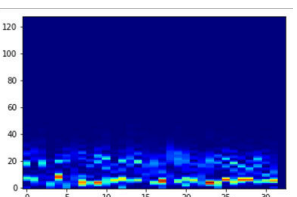

(d)

Fig. 10. Spectrograms of EEG signals from a person suffering an epileptic seizure. Window length $=128$ and $0 \%$ overlapping(a) Rectangular, (b) Gaussian, (c) Blackman and (d) Hanning.

From the previous spectrograms, it can be seen that the data is better displayed in narrow or long windows. In these cases, narrow windows are ideal for the EEG signals from healthy people and longer windows for the signals from patients experiencing epileptic seizures. 
Parameter Experimentation for Epileptic Seizure Detection in EEG Signals using Short-Time...

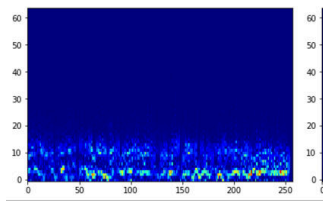

(a)

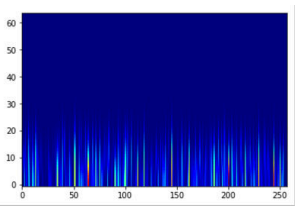

(b)
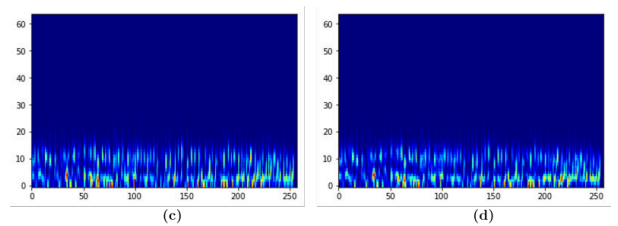

Fig. 11. Spectrograms of EEG signals from a person suffering an epileptic seizure. Window length $=64$ and $75 \%$ overlapping(a) Rectangular, (b) Gaussian , (c) Blackman and (d) Hanning.
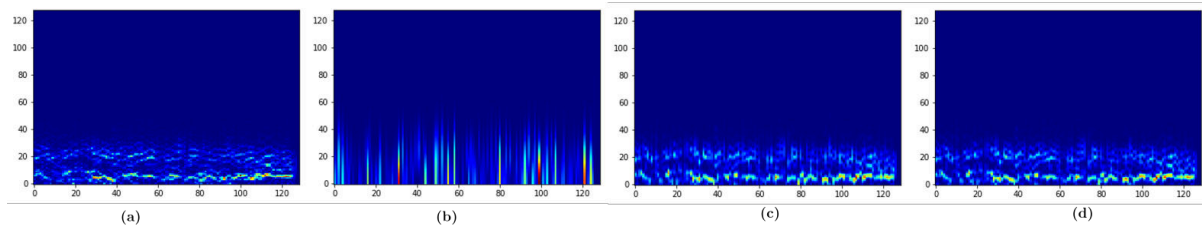

Fig. 12. Spectrograms of EEG signals from a person suffering an epileptic seizure. Window length $=128$ and $75 \%$ overlapping(a) Rectangular, (b) Gaussian, (c) Blackman and (d) Hanning.

\subsection{Classification}

EEG signals were used to generate the spectrograms. Note that the energy is distributed over time for EEG signals from healthy people, while for a person suffering an epileptic attack, the energy expands along the frequency. Based on this observation, windows could be generated for each phenomenon, as mentioned above. As part of the results, the proposed volume-based methodology was used to extract features which were then evaluated by means of classification algorithms, where the algorithm parameters were selected experimentally; the results are shown in Table 1, where the first column shows the windows used and the second describes the parameters, the first parameter is the size of the window separated by a comma from the overlapping.

Different algorithms were used in the evaluation, such as multilayer perceptron (MLP), linear and polynomial support vector machines (SVM), and 3nearest neighbors (KNN); this are shown in columns three through six; for this, a 5 -fold cross-validation was implemented, and the results are shown in terms of accuracy percentage, i.e., the average from both correctly classified classes. For MLP it had two layers with 100 units each one and 200 epochs, this classifier does not reach the highest results, although its performance reach the most with greater than or equal to $95 \%$. Linear SVM seems to have the best results for all the windows, obtaining $99 \%$ with a narrow Gaussian window and overlapping. KNN had a performance similar to linear SVM, with an accuracy of $97.7 \%$ with the same $99 \%$ parameter. Finally, the performance of the polynomial SVM was not as high in relation to all windows; however, it achieved a higher accuracy than KNN, with $98.3 \%$ for one case with a Gaussian window. 
Table 1 shows the highest accuracy for the Gaussian window; the characteristics of this window make it the most balanced in terms of time-frequency resolution due to the similarity of its spectrum to the window; the results were lower for Blackman and Hanning windows, due to their characteristics, although they are variants of the raised cosine and have a spectrum similar to their windows; as in a Gaussian window, this result could be caused by the overlap. Finally, the rectangular window has the lowest results; this does not mean that it is the worst, but rather that it was not useful for this application. This experiment was an initial phase of a complete analysis, which will be carried out in more depth with other parameters in order explore their impact on the classification process, adjust them to find the best classifications, and show how they influence the feature extraction phase in a formal training process.

Table 1. Accuracy results from healthy people versus people suffering epileptic seizures, using four different length windows and two overlapping percentages, with four classifiers.

\begin{tabular}{cccccc}
\hline Window & $\begin{array}{c}\text { Parameters } \\
\text { (size length, } \\
\text { ovelapping) }\end{array}$ & $\begin{array}{c}\text { MLP } \\
\text { (\%) }\end{array}$ & $\begin{array}{c}\text { Linear Polynomial } \\
\text { (\%) }\end{array}$ & $\begin{array}{c}\text { S-NN } \\
\text { (\%) }\end{array}$ & \\
\hline Rectangular & 64,0 & 84.5 & 89.7 & 83.7 & 86.7 \\
\hline Rectangular & 128,0 & 88 & 89.3 & 88.3 & 88.3 \\
\hline Rectangular & 64,48 & 86 & 87.7 & 84.3 & 88.7 \\
\hline Rectangular & 128,96 & 86.5 & 79.6 & 56.6 & 78.3 \\
\hline Gaussian & 64,0 & $\mathbf{9 6 . 5}$ & $\mathbf{9 6 . 3}$ & $\mathbf{9 5 . 7}$ & $\mathbf{9 6 . 7}$ \\
\hline Gaussian & 128,0 & 94.5 & 94 & 91.3 & 91 \\
\hline Gaussian & 64,48 & $\mathbf{9 7}$ & $\mathbf{9 9}$ & $\mathbf{9 8 . 3}$ & $\mathbf{9 7 . 7}$ \\
\hline Gaussian & 128,96 & $\mathbf{9 6 . 6}$ & $\mathbf{9 6 . 6}$ & 88.3 & 96.3 \\
\hline Blackman & $92.5,0$ & 88.3 & 88.3 & 90.7 & 92.3 \\
\hline Blackman & 128,0 & 90.5 & 91.6 & 86.6 & 88.3 \\
\hline Blackman & 64,48 & 93.5 & $\mathbf{9 6 . 7}$ & 86.6 & $\mathbf{9 5 . 7}$ \\
\hline Blackman & 128,96 & $\mathbf{9 5 . 5}$ & 88.7 & 75.3 & 90 \\
\hline Hanning & 64,0 & 93 & 90.3 & 90.7 & 93 \\
\hline Hanning & 128,0 & 87 & 91.6 & 89 & 88.7 \\
\hline Hanning & 64,48 & $\mathbf{9 5}$ & $\mathbf{9 6 . 6}$ & 91 & $\mathbf{9 5 . 3}$ \\
\hline Hanning & 128,96 & $\mathbf{9 5 . 5}$ & 89.3 & 77.3 & 89.3 \\
\hline
\end{tabular}

The reported results were obtained from a general experimentation using some STFT parameters, however, other parameters can be analysed as the energy percentage of the main lobe in relation with side lobes, and the overlapping percentage; whether the parameters proposed are applied to solve another problem the performance will depend of the feature extraction, due to the experimentation show the best results beginning with upper and lower bounds and also the overlapping percentages that best fit the windows. 
Parameter Experimentation for Epileptic Seizure Detection in EEG Signals using Short-Time...

\section{Conclusions}

In several works about EEG analysis, different parameters values are fixed in the STFT but they are neither tested nor justified. From a brief theoretical analysis and some experiments, it can be seen that these parameters are important, as they affect the spectrogram. In particular, the width of the window and the overlap of the signal must be taken into account. A good choice of window could be Blackman, Hanning, Haming, or Gaussian, due to their properties and spectral behaviors. Gaussian window showed the best performance, and the next were Blackman and Hanning; rectangular window was the lowest in the classification, our experiments are based on peaks, thus, probably secondary lobes is affecting the main lobe, which peak is obtained.

Due to the energy shown in the spectrograms, a window should probably be proposed for each type of class, since the windows require different lengths if energy is shown over time or frequency. In this paper, we noted that different parameter affect spectrogram and performance, hence, these have to be selected with a previous analysis, an experimental analysis could be not enough.

As future work, other data sets will be analyzed, since only two signals from two classes from the epilepsy data database were analyzed in these experiments. The experimentation in this paper for different parameters of the STFT was very general and in future experimentation we consider to analyze the windows with respect to spectrum, energy percentage, lobes, and other factors. The energy percentage of the main lobe with respect to the side lobes could be a good parameter. In addition window lengths will be proposed based on the signal frequencies.

Acknowledgments. The first author thanks the support from CONACyTMexico through the scholarship number 457637.

\section{References}

1. Alçin, Ö.F., Siuly, S., Bajaj, V., Guo, Y., Şengür, A., Zhang, Y.: Multi-category eeg signal classification developing time-frequency texture features based fisher vector encoding method. Neurocomputing 218, 251 - 258 (2016)

2. Andrzejak, R.G., Lehnertz, K., Mormann, F., Rieke, C., David, P., Elger, C.E.: Indications of nonlinear deterministic and finite-dimensional structures in time series of brain electrical activity: Dependence on recording region and brain state. Phys. Rev. E 64, 1-8 (2001)

3. Gandhi, V.: Chapter 2 - interfacing brain and machine. In: Gandhi, V. (ed.) BrainComputer Interfacing for Assistive Robotics, pp. 7 - 63. Academic Press, San Diego (2015)

4. Goodwin, M.M.: The STFT, Sinusoidal Models, and Speech Modification, pp. 229258. Springer Berlin Heidelberg, Berlin, Heidelberg (2008)

5. Grossi, E., Olivieri, C., Buscema, M.: Diagnosis of autism through eeg processed by advanced computational algorithms: A pilot study. Computer Methods and Programs in Biomedicine 142, 73 - 79 (2017) 
6. Ilyas, M.Z., Saad, P., Ahmad, M.I.: A survey of analysis and classification of eeg signals for brain-computer interfaces. In: 2015 2nd International Conference on Biomedical Engineering (ICoBE). pp. 1-6 (2015)

7. Kovacs, P., Samiee, K., Gabbouj, M.: On application of rational discrete short time fourier transform in epileptic seizure classification. In: 2014 IEEE International Conference on Acoustics, Speech and Signal Processing (ICASSP). pp. 5839-5843 (2014)

8. Kumar, J.S., Bhuvaneswari, P.: Analysis of electroencephalography (eeg) signals and its categorizationa study. Procedia Engineering 38, 2525 - 2536 (2012), international Conference on Modeling Optimization and Computing

9. Mateo, C., Talavera, J.A.: Short-time fourier transform with the window size fixed in the frequency domain. Digital Signal Processing 77, 13 - 21 (2018), digital Signal Processing and SoftwareX - Joint Special Issue on Reproducible Research in Signal Processing

10. Mustafa, M., Taib, M.N., Murat, Z.H., Hamid, N.H.A.: Glcm texture classification for eeg spectrogram image. In: 2010 IEEE EMBS Conference on Biomedical Engineering and Sciences (IECBES). pp. 373-376 (2010)

11. Prabhu, K.: Window Functions and Their Applications in Signal Processing. CRC Press (2013)

12. Ramos, R., Olvera-López, J.A., Olmos, I.: Analysis of EEG signal processing techniques based on spectrograms. Research in Computing Science 145, 151-162 (2017)

13. Ridouh, A., Boutana, D., Bourennane, S.: Eeg signals classification based on time frequency analysis. Journal of Circuits, Systems and Computers 26(12), 1-26 (2017)

14. Şengür, A., Guo, Y., Akbulut, Y.: Time-frequency texture descriptors of eeg signals for efficient detection of epileptic seizure. Brain Informatics 3(2), 101-108 (2016)

15. Sethares, W.A.: Rhythm and Transforms. Springer London, London (2007)

16. Siuly, S., Li, Y., Zhang, Y.: Electroencephalogram (EEG) and Its Background, pp. 3-21. Springer International Publishing, Cham (2016) 